\title{
In situ magnetotail magnetic flux calculation
}

\author{
M. A. Shukhtina and E. Gordeev \\ St. Petersburg State University, Earth Physics Department, Ulyanovskaya 1, Petrodvoretz, St. Petersburg 198504, Russia \\ Correspondence to: M. A. Shukhtina (mshukht@geo.phys.spbu.ru)
}

Received: 13 October 2014 - Revised: 17 May 2015 - Accepted: 26 May 2015 - Published: 18 June 2015

\begin{abstract}
We explore two new modifications of the magnetotail magnetic flux $(F)$ calculation algorithm based on the Petrinec and Russell (1996) (PR96) approach of the tail radius determination. Unlike in the PR96 model, the tail radius value is calculated at each time step based on simultaneous magnetotail and solar wind observations. Our former algorithm, described in Shukhtina et al. (2009), required that the "tail approximation" requirement were fulfilled, i.e., it could be applied only tailward $x \sim-15 R_{E}$. The new modifications take into account the approximate uniformity of the magnetic field of external sources in the near and middle tail. Tests, based on magnetohydrodynamics (MHD) simulations, show that this approach may be applied at smaller distances, up to $x \sim-3 R_{E}$. The tests also show that the algorithm fails during long periods of strong positive interplanetary magnetic field (IMF) $B_{z}$. A new empirical formula has also been obtained for the tail radius at the terminator (at $x=0$ ) which improves the calculations.
\end{abstract}

Keywords. Magnetospheric physics (magnetotail)

\section{Introduction}

Magnetotail magnetic flux is one of the key parameters in magnetospheric physics (Russell and McPherron, 1973). However, this global parameter is difficult to measure using local observations. Only about 2 decades ago did the global auroral images of Polar and Image spacecraft become available, providing the opportunity to calculate the polar cap (PC) area (Brittnacher et al., 1999; Milan et al., 2003; DeJong and Clauer, 2005; Hubert et al., 2006). As the lobe magnetic field threads the polar caps, the PC area is proportional to the open magnetic flux. However, the PC area determination has its own problems, associated with the threshold level of the registered luminosity and with dayglow contamination. Note that since 2006 there have been no space mis- sions with the purpose of global auroral imaging. Another method of PC area determination is based on identifying the location of region 1 field-aligned currents in global estimates of the Birkeland current system provided by the Active Magnetosphere and Planetary Electrodynamics Response Experiment (AMPERE) (Clausen et al., 2012).

Petrinec and Russell (1996) proposed another approach to the magnetotail magnetic flux calculation. Their method is based on the idea of a flaring magnetopause and on the pressure balance at the magnetopause:

$0.88 P_{\mathrm{d}} \sin ^{2} \alpha+B_{\mathrm{sw}}^{2} / 2 \mu_{0}+n_{\mathrm{sw}} k T_{\mathrm{sw}}=B_{L}^{2} / 2 \mu_{0}$,

where $P_{\mathrm{d}}$ is the solar wind dynamic pressure, $B_{\mathrm{sw}}$ is the interplanetary magnetic field magnitude and $n_{\mathrm{sw}}$ and $T_{\mathrm{sw}}$ are the observed solar wind plasma density and temperature. Assuming the pressure balance in the tail, we hereafter use the equivalent tail lobe magnetic field, determined from the equation

$B_{L}^{2} / 2 \mu_{0}=B^{2} / 2 \mu_{0}+n k T$.

Based on a large data set of simultaneous solar wind and tail lobe measurements, PR96 constructed an empirical model, giving the magnetotail radius $R_{T}$ value as a function of solar wind dynamic pressure $P_{\mathrm{d}}$, interplanetary magnetic field (IMF) $B_{z}$ and an $x$ coordinate in the tail. However, the magnetotail radius value depends not only on the solar wind/IMF parameters, but also on the magnetospheric state. Thus Shukhtina et al. (2009) proposed a modification of the PR96 method, in which the radius is calculated based on in situ measured lobe field (or total pressure) values. Following PR96, the method presumes magnetopause flaring, so that $\tan \alpha=\mathrm{d} R_{T} / \mathrm{d} x$, where $\alpha$ is the flaring angle of the axially symmetric magnetopause (see Fig. 1 adapted from Fig. 2 of PR96). Thus $R_{T}(x)=R_{T 0}+\int_{0}^{x} \tan \alpha(x) \mathrm{d} x$ with $R_{T 0}$ for the 


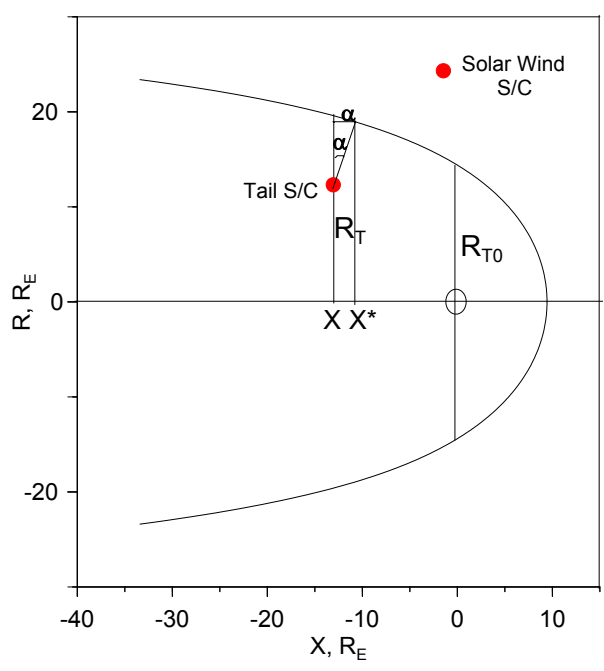

Figure 1. The scheme, illustrating the procedure of $R_{T}$ calculation.

radius value at terminator (at $x=0$ ). As in PR96, the $\alpha(x)$ dependence is taken as $\sin ^{2} \alpha=A^{2} \exp (C x)$. According to the statistical study by Shukhtina et al. (2004), the average $C$ value is $C=0.0714$. All formulas are presented in the geocentric solar magnetospheric (GSM) coordinate system.

Assuming $B_{L}$ isolines perpendicular to magnetopause, a correction $\Delta x$ should be made for the $x$ value at magnetopause, $\Delta x=\left(R_{T}-\left(y^{2}+z^{2}\right)^{1 / 2}\right) \sin \alpha \cos \alpha$, where $x, y, z$ are the coordinates of the observation point. So the $x$ value at the magnetopause, corresponding to the given $B_{L}$, is $x^{*}=$ $x+\Delta x$, giving a new $A^{*}$ value in the $\sin ^{2} \alpha(x)$ dependence, etc. After several iterations the procedure quickly converges, and finally,

$R_{T}(x)=R_{T 0}-2 / C\left(\arcsin \left(A^{*} \exp (C x / 2)\right)-\arcsin \left(A^{*}\right)\right)$.

According to PR96:

$R_{T 0}=14.63\left(P_{\mathrm{d}} / 2.1\right)^{-1 / 6}$.

In the present study this empirical dependence is reexamined, and now it looks as follows:

$R_{T 0}=16.37 P_{\mathrm{d}}^{-1 / 6.02}\left(1.004-0.0054 \mathrm{IMF} B_{z}\right)$.

Formula (2b) will be explained in more detail in Sect. 4 and in the Appendix. As is shown there, formula (2b) suits the tests made in this study better than (2a), so hereafter we everywhere use it in our calculations.

Finally, the magnetic flux value is calculated as follows:

$F_{T}=0.5 \pi R_{T}^{2} B_{L}$,

assuming $B_{L}$ uniformity in the given cross section; the plasma sheet width is neglected.
The algorithm is described in more detail in Shukhtina et al. (2009), where it was tested by global magnetohydrodynamics (GMHD) simulations using the Open Geospace General Circulation Model model (Raeder et al., 2008). Comparison of the algorithm with the imager-based methods of polar cap area estimate is presented in Shukhtina et al. (2010). Both MHD tests and comparison with polar cap images give rather good results during substorm growth phase and steady magnetospheric convection (SMC) events, but the result is worse after substorm onset (especially when the spacecraft is in the plasma sheet) due to the break of the one-dimensional pressure balance. According to both tests the $F_{T}$ values, calculated by the algorithm, exceed those given by direct integration in MHD simulation and those, obtained from auroral images, by $\sim 0.2 \mathrm{GWb}$, which is partly due to ignoring the plasma sheet thickness.

As the algorithm is based on pressure balance, it was applied in the region where the "tail approximation" is valid, i.e., approximately at $x<-15 R_{E}$. It is however desirable to apply the algorithm to spacecraft measurements earthward from $-15 R_{E}$, which would significantly extend the ability to monitor the tail flux. This is the aim of the present work.

All comparisons are based on GMHD simulations made in the Community Coordinated Modeling Center (CCMC; http: //ccmc.gsfc.nasa.gov/). In spite of the well-known problems mainly due to absence of kinetic effects, the first-principlebased GMHD approach provides a reasonable global configuration and dynamics of magnetospheric system (Kaymaz et al., 1995; Gordeev et al., 2013). We believe such tests are perhaps the most straightforward and reliable at present, as they provide an opportunity to calculate the reference magnetotail flux value with high precision for further comparison with our estimates.

\section{Algorithm modifications}

To broaden the working area, we make further modifications to the method. Notice that the open magnetic flux is formed by the "external" part of magnetic field $\boldsymbol{B}_{\mathrm{ext}}=$ $\boldsymbol{B}-\boldsymbol{B}_{\mathrm{IGRF}}$. Unlike the strongly non-uniform dipole field, the external field is approximately uniform and thus may be determined from a measurement in a single point. It is illustrated by Fig. 2 which shows the $\boldsymbol{B}_{\text {ext }}$ field lines and $\boldsymbol{B}_{\text {ext }}$ magnitude distribution (by color) together with total pressure $\left(B_{L}\right)$ isolines and magnetopause position before $(a, t=255 \mathrm{~min})$ and after $(b, t=280 \mathrm{~min})$ substorm onset for the cross section $y=0$ for the MHD simulation BATSRUS_Gordeev_110309_1. The substorm onset is defined here as the magnetotail flux unloading (Fig. 3). The magnetopause (fluopause, see Palmroth et al., 2003), obtained from the simulation data, is in black, whereas the magnetopause, built according to Eq. (2) for the virtual spacecraft with coordinates $x=[-25: 0] R_{E}$ (with a step $1 R_{E}$ ), $y=0, z=11$ $R_{E}$ is designated by red triangles. Figure 2 demonstrates that (1) the $B_{L}$ isolines are approximately perpendicular to mag- 

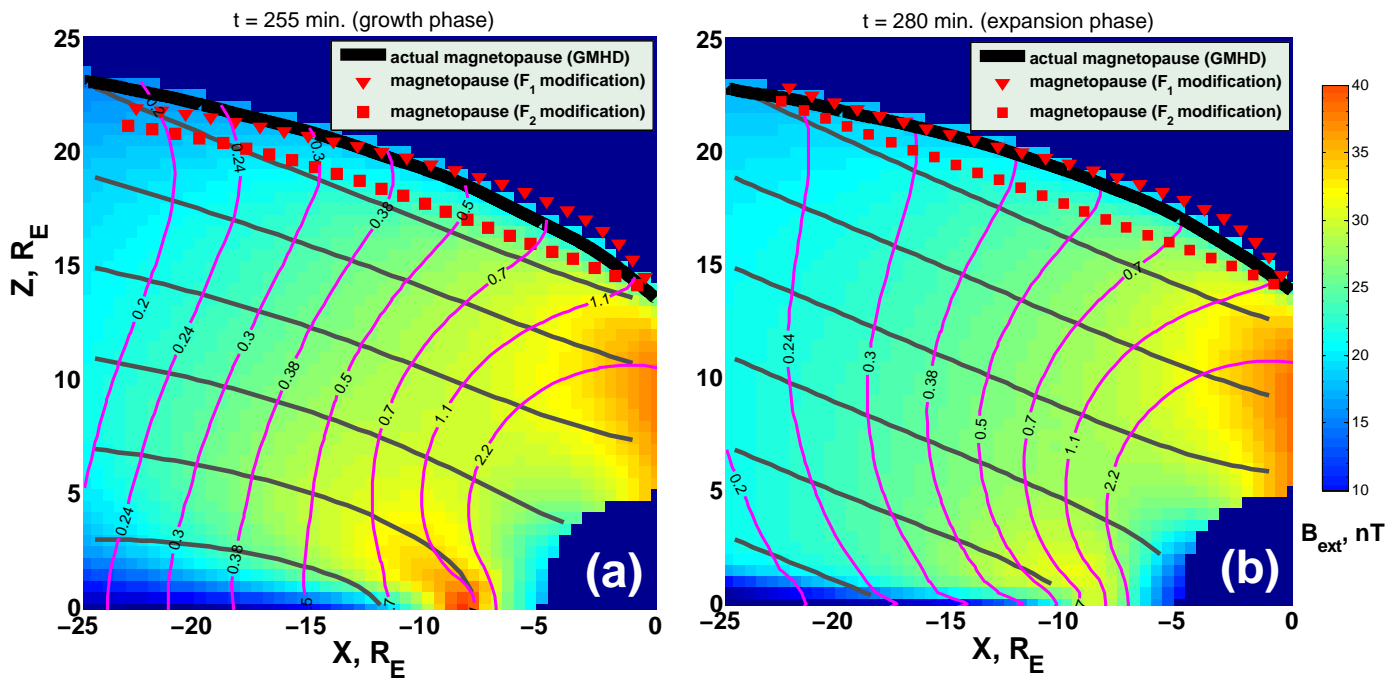

Figure 2. Results of the simulation BATSRUS_Gordeev_110309_1 in the meridional magnetosphere cross section $y=0$ for two simulation

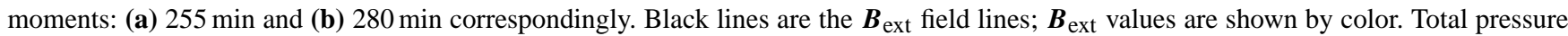
(in $\mathrm{nPa}$ ) isolines (magenta) are also shown. The solid black line indicates the reference magnetopause (fluopause), whereas the red triangles and red squares designate the $R_{T}$ and $R_{T \text { ext }}$ position.

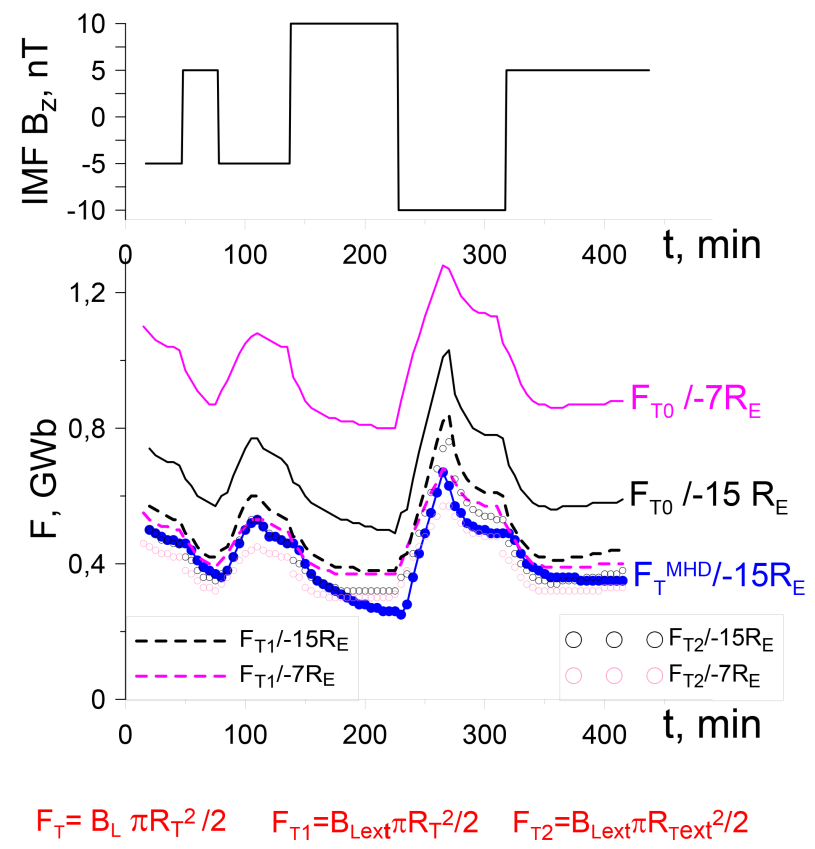

Figure 3. Results of the simulation BATSRUS_Gordeev_110309_1. Top panel: IMF $B_{z}$ variation, shifted by convection to $x=-15 R_{E}$. Bottom panel: $F_{T 0}$ (solid lines), $F_{T 1}$ (dashed lines) and $F_{T 2}$ (circles) values, calculated based on the "measurements" at different $x$ distances $\left(y=0, z=-10 R_{E}\right)$ compared with $F_{T}^{\mathrm{MHD}}$ value, obtained by direct integration through the cross section $x=-15 R_{E}$ (thick blue line).

netopause in the tail lobes at $\sim x<-10 R_{E}$, justifying the $R_{T}$ calculation procedure for this domain; (2) the "external magnetic field" is approximately uniform in the entire volume of the tail lobes up to $x \sim-2 \div-5 R_{E}$, its isolines being perpendicular to magnetopause in this region.

Analogously to $B_{L}$ we introduce the quantity $B_{L \text { ext }}$ :

$B_{L \text { ext }}^{2} / 2 \mu_{0}=\left(B_{\text {ext }}\right)^{2} / 2 \mu_{0}+n k T$,

which is also approximately uniform in a vast volume of the magnetotail. Then the proxy of the magnetic flux of external sources is

$F_{T 1}=0.5 \pi R_{T}^{2} B_{L \mathrm{ext}}$.

Note that the procedure of $B_{L \text { ext }}$ calculation is reasonable only if the vectors $\boldsymbol{B}_{\mathrm{IGRF}}$ and $\boldsymbol{B}_{\text {ext }}$ are more or less collinear. So $B_{L \text { ext }}$ is defined only if the angle between $\boldsymbol{B}_{\text {IGRF }}$ and $\boldsymbol{B}_{\text {ext }}$ is less than $90^{\circ}$. Otherwise (e.g., in the plasma sheet, where $\boldsymbol{B}_{\text {ext }}$ and $\boldsymbol{B}_{\text {IGRF }}$ are antiparallel) $B_{L \text { ext }}$ is not defined. Most correctly (4) and the following formula (5) may be applied for the observations in the tail lobes.

However, the usage of the $F_{1}$ proxy faces some difficulties. Note that Eq. (2) contains arcsin functions and cannot be applied when the arcsin argument exceeds 1. Furthermore, for the points in the inner tail the "corrected" value $x^{*}$ may become positive (the observation point "moves" to the dayside). According to our experience, this situation is not infrequent in the near-Earth region with large $\boldsymbol{B}$ values. To avoid this difficulty, we attempt one more modification of the algorithm. In the right-hand side of expression (1) instead of $B_{L}$ we put $B_{L \text { ext }}$. As pointed above, the $B_{L \text { ext }}$ isolines (perpendicular in the lobes to the field lines of external field) 
are approximately perpendicular to the magnetopause in a more vast region (including the inner magnetotail) than the $B_{L}$ isolines. So the procedure of radius calculation may be applied to the $B_{L \text { ext }}$ quantity instead of $B_{L}$ up to $\sim-5 R_{E}$. The surface, corresponding to the obtained radius, is not the real magnetopause, but is a surface inside it, with the radius $R_{T \text { ext }}$. The proxy of the open magnetic flux confined by this surface is now

$F_{T 2}=0.5 \pi R_{T \text { ext }}^{2} B_{L \mathrm{ext}}$.

The magnetopause proxy, corresponding to the $R_{T \text { ext }}$ value, is shown in Fig. 2 by red squares. For snapshots in Fig. 2, earthward from $-15 R_{E}$ the $R_{T}$ value is slightly larger than the fluopause radius, whereas the $R_{T \text { ext }}$ value is smaller. The actual magnetopause lies between $R_{T}$ and $R_{T \text { ext }}$. Tailward from $-15 R_{E}$ the $R_{T}$ value is close to the actual radius, whereas $R_{T \text { ext }}$ lies inside it. At $\sim-23 R_{E}$ both estimates become identical.

So we have three magnetotail magnetic flux proxies, $F_{T 0}$ (according to Eq. 3), $F_{T 1}$ (Eq. 4) and $F_{T 2}$ (Eq. 5). Note that all of them assume the lobe field to be uniform across the whole tail cross section, neglecting the plasma sheet thickness. At the same time the $F_{T 2}$ algorithm removes a part of the magnetic flux, which offsets the excess flux in the plasma sheet.

The quantities that may be calculated in the MHD simulations are $F_{T}^{\mathrm{MHD}}$ - the total magnetic flux through the given tail cross section and $F_{\mathrm{PC}}^{\mathrm{MHD}}$ - the magnetic flux, formed by the open lobe magnetic field lines. In the middle tail (e.g., at $x=-15 R_{E}$ ) these quantities should be close to each other, the $F_{T}^{\mathrm{MHD}}$ value being slightly larger. We expect our proxies $F_{T 1}$ and $F_{T 2}$ to lie somewhere between them, $F_{T 2}$ being closer to $F_{\mathrm{PC}}^{\mathrm{MHD}}$. The algorithm of $F_{T 0}$ calculation was examined in Shukhtina et al. $(2009,2010)$. The $F_{T 1}$ and $F_{T 2}$ calculation is less strict and requires thorough testing. These tests are presented below.

\section{Evaluation of the method}

In this section we present the results of the $F_{T 0}, F_{T 1}$ and $F_{T 2}$ comparison with the $F_{T}^{\mathrm{MHD}}$ values, obtained by direct magnetic flux integration through the magnetotail cross section $x=-15 R_{E}$ as described in Gordeev et al. (2011). The comparison was carried out for two global MHD BATS-R-US simulations (Powell et al., 1999): with synthetic SW input (artificial event) and with real SW input (real event). For the second simulation, we also consider the polar cap magnetic flux $F_{\mathrm{PC}}^{\mathrm{MHD}}$ quantity, available from the CCMC website.

\subsection{Artificial event (event 1)}

Figure 3 presents results based on the simulation BATSRUS_Gordeev_110309_1, which was already presented in Fig. 2. The only variable input parameter IMF $B_{z}$ (shifted by convection time from $x=33 R_{E}$ to $x=$ $-15 R_{E}$ ) is shown in the top panel, all other inputs being fixed: IMF $B_{x}=\mathrm{IMF} B_{y}=0$, solar wind speed $V_{x}=$ $-300 \mathrm{~km} \mathrm{~s}^{-1}, V_{y}=V_{z}=0$, ion density $N=20 \mathrm{~cm}^{-3}$ and temperature $T=100000 \mathrm{~K}$, zero dipole tilt. The bottom panel presents the directly integrated magnetotail magnetic flux $F_{T}^{\mathrm{MHD}}$ (thick blue line) together with $F_{T 0}$ estimates based on "measurements" of the virtual spacecraft at $x=$ $-15 R_{E}$ and $-7 R_{E}\left(y=0, z=-10 R_{E}\right)$. The $F_{T 0}$ quantity behaves similarly to $F_{T}^{\mathrm{MHD}}$, but there is an offset, which grows when approaching the Earth (at $x=-7 R_{E}$ the $F_{T 0}$ value is twice as large as $F_{T}^{\mathrm{MHD}}$ ). Dashed lines of the corresponding color indicate the $F_{T 1}$ values for the same virtual spacecraft positions, whereas circles correspond to $F_{T 2}$. In contrast to $F_{T 0}$, the $F_{T 1}$ and $F_{T 2}$ values for different observation points are close to each other and to the $F_{T}^{\mathrm{MHD}}$ value, on average bounding it. So $F_{T 1}$ and $F_{T 2}$ may be considered as the upper and lower $F_{T}^{\mathrm{MHD}}$ estimates. Note that the worst correspondence is observed during the long ( $\sim 90 \mathrm{~min})$ period of IMF $B_{z}=+10 \mathrm{nT}$, where all estimates do not follow $F_{T}^{\mathrm{MHD}}$ and exceed it. We shall return to analysis of this effect in Sect. 3.2.

Figure 3 shows that the $F_{T 0}$ proxy is not appropriate inside $-15 R_{E}$, so in the next section we test only the $F_{T 1}$ and $F_{T 2}$ proxies on a more interesting simulation, reproducing a real geophysical event.

\subsection{Real event (event 2)}

Another CCMC simulation studied is BATSRUS_Sergeev_060508_1. The input parameters (including the dipole tilt) were taken from a real event, 00:00-16:00 UT 5 March 2008 (Fig. 4). The event includes different geomagnetic situations with variable IMF $B_{z} ; P_{\mathrm{d}}$ smoothly changes between $\sim 1$ and $3 \mathrm{nPa}$. The laborious $F_{T}^{\mathrm{MHD}}$ calculations were done for the period 09:00-16:00 UT, whereas the polar cap magnetic flux value $F_{\mathrm{PC}}^{\mathrm{MHD}}$, formed by the open field lines, is presented at the CCMC website for the whole simulation period.

Figure 5a presents the "merging electric field" $E_{\mathrm{m}}$ variation for the period studied. Here $E_{\mathrm{m}}=V B_{t} \sin ^{3}(\Theta / 2)$, where $B_{t}=\sqrt{\left(\operatorname{IMF} B_{y}\right)^{2}+\left(\operatorname{IMF} B_{z}\right)^{2}}, V$ is the solar wind velocity and $\Theta$ is the clock angle (see, e.g., Boyle et al., 1997). Solar wind/IMF parameters are again shifted to $x=$ $-15 R_{E}$ by convection. Figure $5 \mathrm{~b}$ presents different magnetic flux estimates for the Northern Hemisphere: in green and black - the $F_{T}^{\mathrm{MHD}}$ and $F_{\mathrm{PC}}^{\mathrm{MHD}}$ values, in red and blue - the $F_{T 1}$ and $F_{T 2}$ values correspondingly. The $F_{T 1}$ and $F_{T 2}$ values were calculated based on the "measurements" of a virtual spacecraft at the point $(-15,0,12) R_{E} . F_{T 1}$ values, calculated for different x positions, are presented in Fig. $5 \mathrm{c}$. Variations of all magnetic flux proxies repeat the main features of $E_{\mathrm{m}}$ variations. The $F_{T}^{\mathrm{MHD}}$ and $F_{\mathrm{PC}}^{\mathrm{MHD}}$ curves are close to each other except the period of positive IMF $B_{z}$ af- 


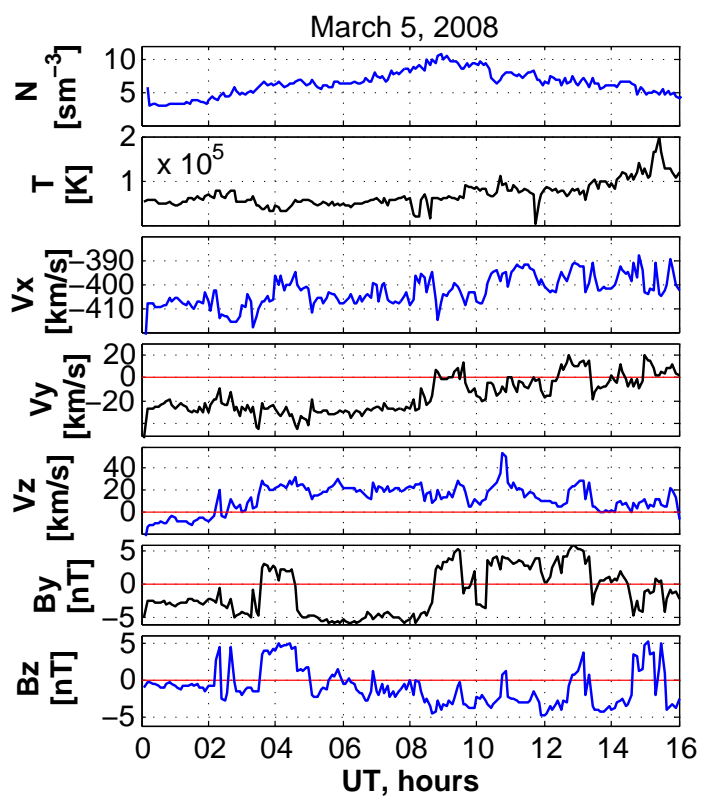

Figure 4. The input parameters of the simulation BATSRUS_Sergeev_060508_1. From top to bottom: ion density $(N)$, ion temperature $(T)$, solar wind speed components $\left(V_{x}, V_{y}, V_{z}\right)$ and IMF components $\left(B_{x}, B_{y}, B_{z}\right)$.

ter 15:00 UT. The regression equation for the period 09:0015:00 UT is $F_{T}^{\mathrm{MHD}}=0.87 F_{\mathrm{PC}}^{\mathrm{MHD}}+0.05$, with correlation coefficient $C C=0.85$, demonstrating cross-validation of the two estimates. Notice however that as $F_{T}^{\mathrm{MHD}}$ is the total flux estimate, and the $F_{\mathrm{PC}}^{\mathrm{MHD}}$ is the open flux proxy, $F_{T}^{\mathrm{MHD}}$ should slightly exceed $F_{\mathrm{PC}}^{\mathrm{MHD}}$. According to Fig. 5 the balance is inverse (average $F_{T}^{\mathrm{MHD}}$ and $F_{\mathrm{PC}}^{\mathrm{MHD}}$ values for the period 09:0015:00 UT are 0.62 and $0.66 \mathrm{GWb}$ correspondingly). Until now we do not know the reason of such inconsistency in the GMHD simulation.

We made a regression analysis of $F_{T 1}$ and $F_{T 2}$ quantities, calculated for different observation points (see the legends in Fig. 5b, c) with both $F_{T}^{\mathrm{MHD}}$ and $F_{\mathrm{PC}}^{\mathrm{MHD}}$ for the period 09:0015:00 UT, when both variables were available. The results appeared to be very close (identical taking into account the error bars). As the $F_{\mathrm{PC}}^{\mathrm{MHD}}$ values are available for the whole period 00:00-16:00 UT, hereafter we compare different magnetic flux proxies with the $F_{\mathrm{PC}}^{\mathrm{MHD}}$ value.

Note that the major discrepancies between algorithm results and $F_{\mathrm{PC}}^{\mathrm{MHD}}$ appear during the periods of zero $E_{\mathrm{m}}$ (strong positive IMF $B_{z}$ ) 03:40-05:40 UT and 15:00-16:00 UT. The same situation was observed in the simulation with artificial input (Fig. 3), i.e., such periods are not adequately described by our algorithms. Thus we exclude these periods (03:4005:40 UT and 15:00-16:00 UT) from our regression analysis, leaving this topic for the future.

According to Fig. 5 the variations and absolute values of $F_{T 1}$ are similar to those of $F_{\mathrm{PC}}^{\mathrm{MHD}}$, whereas the $F_{T 2}$ proxy reproduction of the reference magnetic flux is slightly worse.
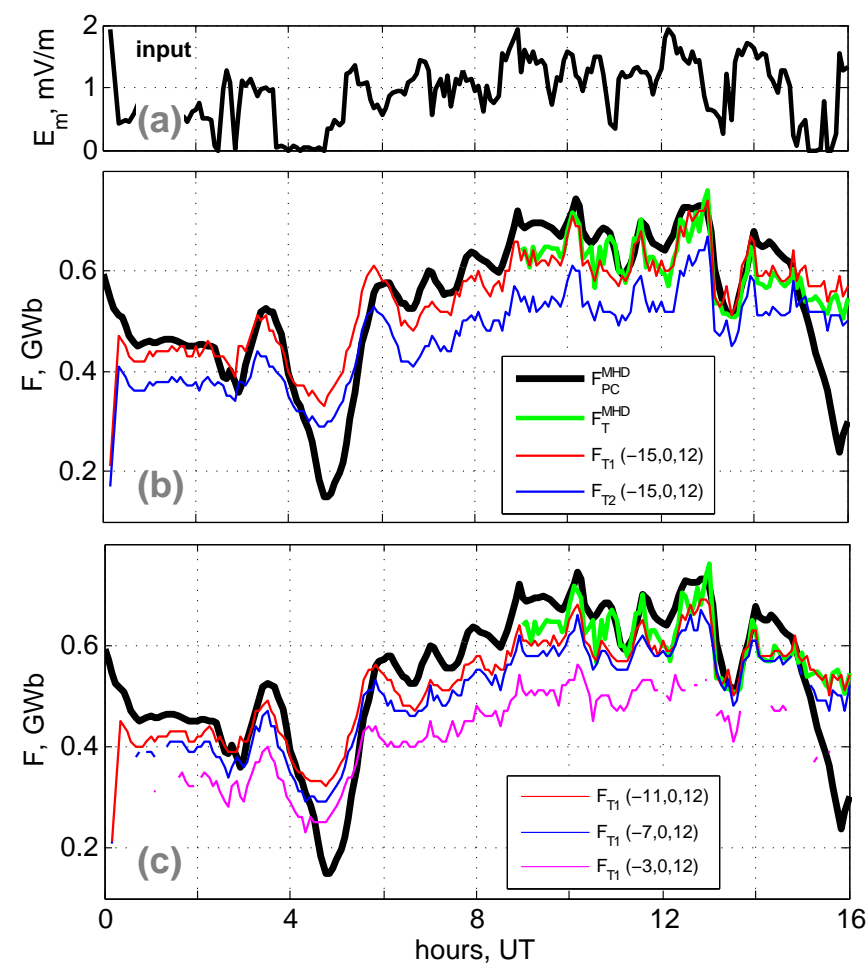

Figure 5. Results of the simulation BATSRUS_Sergeev_060508_1. (a) Solar wind "merging electric field" $E_{\mathrm{m}}$ variation. (b) Different $F$ estimates: $F_{T}^{\mathrm{MHD}}$ (green), $F_{\mathrm{PC}}^{\mathrm{MHD}}$ (black), $F_{T 1}$ (red) and $F_{T 2}$ (blue). The $F_{T 1}$ and $F_{T 2}$ quantities are calculated for the point $(-15,0,12)$. (c) $F_{T 1}$ values, calculated at different distances $\mathrm{x}=-11$ (red), -7 (blue) and $-3 R_{E}$ (magenta), compared with $F_{\mathrm{PC}}^{\mathrm{MHD}}$ (black) and $F_{T}^{\mathrm{MHD}}$ (green).

The figure also demonstrates that the $F_{T 1}$ quantity may serve as a rather stable estimate of the tail magnetic flux for spacecraft positions up to $x=-7 R_{E}$.

However, until now we considered only separate observation points. Below, we discuss the global distribution of different magnetic flux estimates.

\subsection{Global regression analysis for the real event}

Figure 6 presents the global distribution of results of regression analysis of all three $\left(F_{T 0}, F_{T 1}\right.$ and $\left.F_{T 2}\right)$ magnetic flux estimates with $F_{\mathrm{PC}}^{\mathrm{MHD}}$ quantity for the cross section $x=-7 R_{E}$ for the same simulation BATSRUS_Sergeev_060508_1. The solid black line designates the boundary where plasma $\beta=1$.

The comparison is carried out using the equation

$F_{T j}=P_{1} F_{\mathrm{PC}}^{\mathrm{MHD}}+P_{2}, \quad j=0,1,2$.

Figure 6 presents the distribution of the following statistical parameters: average over the simulation $F$ values (upper row), correlation $C C$ (second row) and regression $P_{1}$ (third 

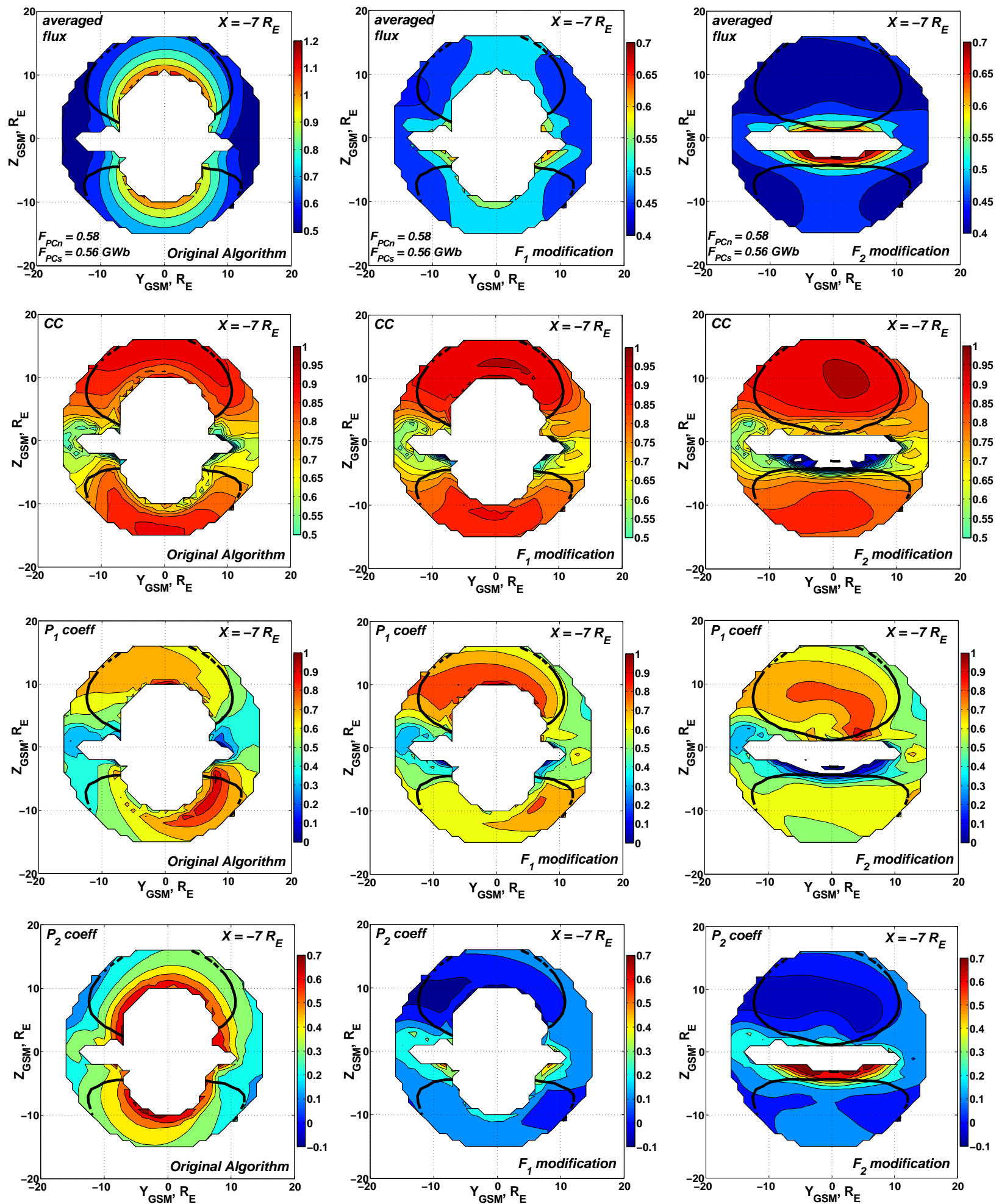

Figure 6. Real event simulation BATSRUS_Sergeev_060508_1. Distributions of the average over the simulation flux value, correlation and regression coefficients and the free term, describing the relationship of $F_{T 0}, F_{T 1}$ and $F_{T 2}$, "measured" at $x=-7 R_{E}$, with the $F_{\mathrm{PC}}^{\mathrm{MHD}}$ value. Black thick contour on each panel bounds the lobes with $\beta<1$. Note the different color bar scale for the average values of $F_{T 0}$ and $F_{T 1}$, $F_{T 2}$ in the first row. 
row) coefficients and the free term $P_{2}$ (bottom row) for all observation points in the cross section $x=-7 R_{E}$ (note that the intervals of large positive IMF $B_{z}$ 03:40-05:40 and 15:0016:00 UT are excluded). The points in the Northern Hemisphere are compared with the northern polar cap flux $F_{\mathrm{PC}}^{\mathrm{MHD}}$, in the Southern Hemisphere - with the southern $F_{\mathrm{PC}}^{\mathrm{MHD}}$.

The most prominent feature of Fig. 6 is the scope of the $F_{T 2}$ algorithm, which covers almost the whole cross section in contrast to the other two proxies.

The upper row shows that the average $F_{\mathrm{PC}}^{\mathrm{MHD}}$ value is best of all reproduced, though slightly underestimated (by $10 \div 15 \%$ ) by $F_{T 1}$, whereas the $F_{T 2}$ proxy is smaller than $F_{\mathrm{PC}}^{\mathrm{MHD}}$ by $20 \div 25 \%$. Note that the average flux distribution as well as other statistical characteristics are very uniform in space for $F_{T 2}$ and rather uniform for $F_{T 1}$. On the contrary the $F_{T 0}$ characteristics strongly depend on the observation point, making their use problematic. The $C C$ values (second row) are mostly $\geq 0.8$ and often $\geq 0.9$ for $\beta \leq 1$ for all three proxies. Again the distributions for $F_{T 1}$ and $F_{T 2}$ are more uniform than those for $F_{T 0}$. The regression coefficient $P_{1}$ (third row) is mostly around $0.7-0.8$ (sometimes decreasing to 0.6 ) for all three proxies. Finally, the free term $P_{2}$ (the bottom row) is small (about $\pm 0.1 \mathrm{GWb}$ ) for $F_{T 1}$ and $F_{T 2}$ proxies compared to the values $0.3-0.5 \mathrm{GWb}$ for $F_{T 0}$.

In summary, the $F_{T 2}$ proxy may be applied almost in the whole tail lobe region and demonstrates uniform parameters' distributions, though the average flux values are underestimated by $\sim 0.1-0.15 \mathrm{GWb}$. The $F_{T 1}$ proxy reproduces the $F_{\mathrm{PC}}^{\mathrm{MHD}}$ value slightly better than $F_{T 2}$ and better than $F_{T 0}$, but its working area (as well as the scope of $F_{T 0}$ ) is much smaller than that of $F_{T 2}$.

\section{Improved formula for the tail radius at terminator}

The magnetopause radius in the terminator plane $\left(R_{T 0}\right)$ is the starting point for the tail radius calculation (Eq. 2) in our method. As the $R_{T 0}$ value strongly influences the tail radius and consequently the magnetic flux value, it is important to use a precise formula for $R_{T 0}$. For today, there is a set of different empirical magnetopause models based on different data sets and mathematical methods. All these models give $R_{T 0}$ in the global magnetopause shape context, leading to different functional forms $R_{T 0}(\mathrm{SW}, \mathrm{IMF})$. To determine this dependence more precisely, we tried to construct an empirical magnetopause model in the specified narrow region - terminator $(x=0)$ plane. For this purpose we used the data set of magnetopause crossings from the GSFC NASA website (ftpbrowser.gsfc.nasa.gov/magnetopause.html). This data set spans the time range 1963-1998 and uses crossings of 18 different satellites that were analyzed by different authors. The data is tagged by $1 \mathrm{~h}$ averaged SW/IMF measurements. To collect a sufficient number of magnetopause crossings, we selected crossings in the vicinity of the terminator plane (namely in the region $-3<x<2 R_{E}$ ) and traced them to $x=0$ using the analytical magnetopause shape given in PR96. This simple procedure enabled us to collect 1192 crossings that further reduced to 1022 points due to cutting off the deep tails of the SW/IMF parameter distributions and the low-latitude crossings. The low-latitude crossings were rejected because of not sufficiently sharp gradients of plasma and magnetic parameters, which lead to a high uncertainty of the magnetopause determination. Next, the coordinates of Southern Hemisphere crossings were mapped to the Northern Hemisphere with the dipole tilt sign inversion assuming the north-south shape invariance. Applying multiple regression analysis to the selected data set, we found a clear and pronounced linear dependence of $R_{T 0}$ on magnetic dipole tilt angle $\Psi$, a power law dependence on solar wind dynamic pressure $P_{\mathrm{d}}$ and weak linear dependence on IMF $B_{z}$. The final empirical formula for $R_{T 0}$ in the Northern Hemisphere is

$$
\begin{gathered}
R_{T 0}=(14.1+0.045 \Psi) \cdot 1.161 P_{\mathrm{d}}^{-1 / 6.02} \\
\cdot\left(1.004-0.0054 \mathrm{IMF} B_{z}\right),
\end{gathered}
$$

for $\Psi$ in degrees, $P_{\mathrm{d}}$ in $\mathrm{nPa}$, IMF $B z$ in nT and $R_{T 0}$ in Earth's radii $R_{E}$.

The formula is valid for all tilt angles in the SW/IMF parameter range $1<P_{\mathrm{d}}<8 \mathrm{nPa}$ and $-6<\mathrm{IMF} B_{z}<6 \mathrm{nT}$. For more details on the derivation of Eq. (7), see the Appendix.

To check whether the new formula improves the result, we compared calculations based on the PR96 terminator radius and on the new $R_{T 0}$ value for the already considered simulation BATSRUS_Sergeev_060508_1 assuming zero dipole tilt in Eq. (7). The tilt was taken as zero because it is the assumption of our $\mathrm{F}$ calculation algorithm. We performed regression analysis, comparing the polar cap magnetic flux value from the simulation $\left(F_{\mathrm{PC}}^{\mathrm{MHD}}\right)$ with the estimated tail magnetic flux in each point of the chosen cross section. The comparison was made for all three algorithm modifications for different distances $\left(x=-25,-15,-11\right.$ and $\left.-7 R_{E}\right)$. For comprehensive comparison, we calculated differences between the values given by new and old formulas of $R_{T 0}$ for the correlation coefficient $C C$, slope $P_{1}$ and free term $P_{2}$ for the aforementioned cross sections. The differences were calculated as follows:

$\triangle C C=C C_{\mathrm{New}}-C C_{\mathrm{PR} 96}$

$\Delta P_{1}=\left|P_{1}(\mathrm{PR} 96)-1\right|-\left|P_{1}(\mathrm{New})-1\right|$

$\Delta P_{2}=P_{2}(\mathrm{PR} 96)-P_{2}(\mathrm{New})$,

to be positive in the case of the flux estimation accuracy increase.

The values from Eq. (8) turned out to be positive for all algorithm modifications, indicating slightly higher accuracy of the flux estimates. So the new formula for the tail radius at terminator is preferable. 
Table 1. Summary for methods application.

\begin{tabular}{|c|c|c|c|c|}
\hline Method & $\begin{array}{l}\text { Application } \\
\text { scope }\end{array}$ & $\begin{array}{l}\text { Estimation } \\
\text { accuracy }\end{array}$ & $\begin{array}{l}\text { Spatial } \\
\text { uniformity }\end{array}$ & Comments \\
\hline$F_{T 0}$ & $x<-15 R_{E}$ & $\begin{array}{l}\text { significantly } \\
\text { overestimated } \\
(30 \div 60 \%)\end{array}$ & $\begin{array}{l}\text { strongly } \\
\text { non-uniform }\end{array}$ & $\begin{array}{l}\text { 1. Suitable only if tail approximation is fulfilled } \\
\text { 2. Coincides with } F_{T 1} \& F_{T 2} \text { in the midtail } \\
\text { 3. Difficult to calibrate }\end{array}$ \\
\hline$F_{T 1}$ & $x<-7 R_{E}$ & $\begin{array}{l}\text { close to the refer- } \\
\text { ence value }( \pm 20 \%)\end{array}$ & $\begin{array}{l}\text { rather } \\
\text { uniform }\end{array}$ & $\begin{array}{l}\text { 1. Applicable up to }-3 R_{E} \text { if possible } \\
\text { 2. Coincides with } F_{T 0} \& F_{T 2} \text { in midtail } \\
\text { 3. Calibration: } F_{T}^{\mathrm{MHD}}=(0.98 \pm 0.01) F_{T 1}, \\
x=-7,-11,-15 R_{E} \\
N=81.418, \quad C C=0.74, \quad \sigma=0.039 \mathrm{GWb} \\
(\sigma \sim 10 \%)\end{array}$ \\
\hline$F_{T 2}$ & $x<-7 R_{E}$ & $\begin{array}{l}\text { close to the refer- } \\
\text { ence value }( \pm 20 \%)\end{array}$ & $\begin{array}{l}\text { highly } \\
\text { uniform }\end{array}$ & $\begin{array}{l}\text { 1. Applicable up to }-3 R_{E} \text { if possible } \\
\text { 2. Coincides with } F_{T 0} \& F_{T 1} \text { in midtail } \\
\text { 3. Calibration: } F_{T}^{\mathrm{MHD}}=(1.12 \pm 0.01) F_{T 2}, \\
x=-7,-11,-15 R_{E} \\
N=95.330, \quad C C=0.72, \quad \sigma=0.037 \mathrm{GWb} \\
(\sigma \sim 10 \%)\end{array}$ \\
\hline
\end{tabular}

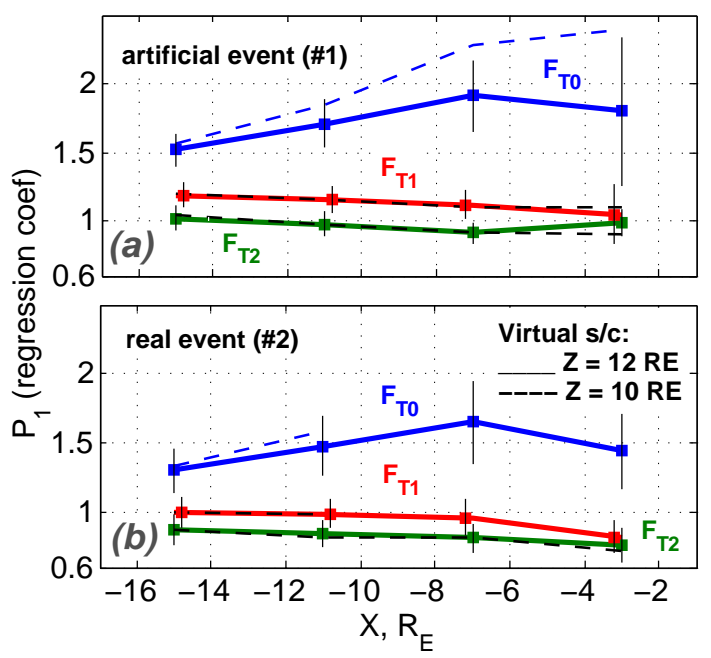

Figure 7. The value of the regression coefficient $P_{1}$ in $F_{T 0(1,2)}=P_{1} F_{T}^{\mathrm{MHD}}$ for different observation points: $x=-15,-11,-7,-3 R_{E}, \quad y=0$ and $z=10,12 R_{E}$. Results are shown for both artificial event (a) and real event (b) simulations. Vertical bars denote the confidence level.

\section{Discussion}

To emphasize the ability of our modified $F_{T 1}$ and $F_{T 2}$ algorithms let us again turn to Fig. 6 . The figure shows that the free term in the regression equations for $F_{T 1}$ and $F_{T 2}$ is fairly small, within $\pm 0.1 \mathrm{GWb}$, and the regression coefficient $P_{1}$ is rather uniform. Similar results were also obtained for other tail cross sections, though there is not enough space to present them. Instead, we briefly illustrate the $P_{1}$ volume uniformity by presenting two x profiles (for $z=10$ and $12 R_{E}$ ) of $P_{1}$ coefficient for each flux estimation method (Fig. 7). Here we use the regression equation assuming zero free term

$F_{T j}=P_{1} F_{T}^{\mathrm{MHD}}, j=0,1,2$.

The results for Events 1 and 2 are presented in panels $\mathrm{a}$ and $\mathrm{b}$ correspondingly. The sampling points are taken at $x=-15,-11,-7,-3 R_{E}, y=0$ and $z=10,12 R_{E}$. Both Fig. 7a and b confirm the high uniformity of regression coefficient corresponding to $F_{T 1}$ and $F_{T 2}$ estimates along the tail (in addition to cross-tail $P_{1}$ uniformity shown in Fig. 6), which slightly varies around unity and has moderate confidence bounds, supporting the idea that the free term in the regression equation can be set to zero. On the contrary, the $P_{1}$ for $F_{T 0}$ estimate is highly non-uniform and has a significantly wider confidence interval due to ignoring the large free term.

High uniformity of the regression coefficient $P_{1}$ and small absolute value of the free term $P_{2}$ in the entire middle- and near-tail lobes give us the opportunity to make a unified calibration of our modified algorithms $F_{T 1}$ and $F_{T 2}$ using simple linear regression with the zero free term for the tail lobes. The calibration formulas are presented in Table 1, summarizing the general results of our analysis. Briefly, in the middle tail $\left(x<-15 R_{E}\right)$, the modifications $F_{T 1}$ and $F_{T 2}$ have values, very close to the former algorithm $F_{T 0}$. Furthermore, in contrast to the original algorithm, the modified algorithms have much wider spatial application limits extended to the inner magnetosphere (especially $F_{T 2}$ ) with highly uniform calibration coefficients. On the basis of the presented analysis, for further applications we recommend using the combination of $F_{T 1}$ and $F_{T 2}$ modifications instead of original method. 
It is important to note that the algorithm presumes zero dipole tilt. In Event 2 the dipole tilt varied between -15 and $5^{\circ}$, which caused the asymmetries in Fig. 6, but still provided a satisfactory result. However, the larger tilt angles can lead to larger errors.

\section{Conclusions}

We present an algorithm for magnetotail magnetic flux $F_{T 1}$ calculation which may be applied for the measurements inside $x=-15 R_{E}$. The algorithm is a modification of the old one (computing the $F_{T 0}$ value), which could be applied tailward $x=-15 R_{E}$. The modified algorithm gives a proxy of the magnetic flux of the external magnetic field (Eq. 4) based on the approximate uniformity of this field. Both algorithms should be preferably applied in the tail lobes, where the 1-D pressure balance is valid according to MHD tests. However, the working area of both $F_{T 0}$ and $F_{T 1}$ estimates is limited because of their dependence on the arcsin function used for the tail radius calculation. To broaden the application domain of the new algorithm, it was again modified (Eq. 5) by using the new radius value $R_{T \text { ext }}$, calculated from the balance of the "external magnetic pressure" (the pressure of the magnetic field of external sources) with the magnetosheath pressure. $R_{T \text { ext }}$ corresponds to a surface, lying inside the actual magnetopause, and is the lower estimate of the tail radius value. According to Fig. 2a and b, $R_{T}$ and $R_{T \text { ext }}$ bound the actual magnetopause. Tests based on two MHD simulations show good $F_{T 1}$ correlation/regression relationships with the magnetic flux value $F_{T}^{\mathrm{MHD}}$, obtained by direct magnetic flux integration through the tail cross section at $x=-15 R_{E}$, and with the magnetic flux of the polar cap $F_{\mathrm{PC}}^{\mathrm{MHD}}$, given by the CCMC. The regressions for $F_{T 2}$ are slightly worse, but the scope of $F_{T 2}$ algorithm is considerably wider than that of $F_{T 1}$; furthermore, the $F_{T 2}$ distribution appeared strongly uniform (weakly depending on the observation point). The quality of all methods degrades for periods of large positive IMF $B_{z}$, when all algorithms give overestimated magnetic flux values.

Also a new empirical dependence for terminator radius $R_{T 0}$, which is the "boundary condition" for our algorithm, was obtained from spacecraft data. We show that the new $R_{T 0}$ formula better represents magnetopause position versus its analogues and should be used in the future. 

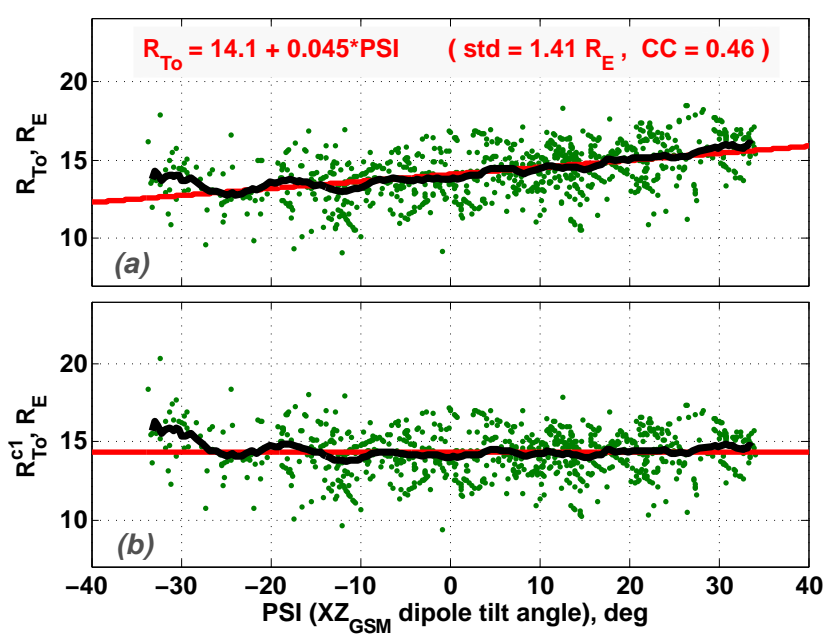

Figure A1. Top panel - scatterplot of terminator radius dependence on dipole tilt angle. Bottom panel - demonstration of results of the data correction procedure (Eq. A2) that eliminates the $R_{T 0}(\Psi)$ dependence. Red straight lines are the linear regression lines. Black lines are the moving averages (window size is $3^{\circ}$, step size is $0.5^{\circ}$ ).

\section{Appendix A: New formulation for the magnetopause radius at terminator (plane $x=0$ )}

\section{A1 Fitting the data}

To find the analytical representation of $R_{T 0}$, we applied the linear regression to three interplanetary medium parameters: Earth's dipole tilt angle $\Psi, \mathrm{SW}$ dynamic pressure $P_{\mathrm{d}}$ and IMF $B_{z}$. First we extracted the $R_{T 0}$ dependence on $\Psi$ (Fig. A1, top panel):

$R_{T 0}=(14.1 \pm 0.1)+(0.045 \pm 0.005) \Psi$.

The figure demonstrates significant $R_{T 0}(\Psi)$ dependence with amplitude $\sim 3 R_{E}$ between extreme angles $\left(\sim \pm 30^{\circ}\right)$ in good agreement with recent models (Lin et al., 2010; Wang et al., 2013) [hereafter L10 and W13]. It is interesting to note the small positive offset of $R_{T 0}$ for large negative tilt angles ( $\Psi<-27^{\circ}$, negative $\Psi$ corresponding to anti-sunward tilt of dipole axis in the Northern Hemisphere) which may be associated with passage of the sunward edge of cusp indentation across the terminator plane (see Fig. 8 in W13, or Fig. 8d in L10).

Since we obtained a clear and strong $R_{T 0}(\Psi)$ dependence, the dipole tilt influence can be excluded from the following analysis using the correction

$R_{T 0}^{c 1}=\frac{R_{T 0} \text { (measured) }}{R_{T 0}(\Psi)} \cdot R_{T 0}^{\mathrm{avg}}$,

where $R_{T 0}$ in the numerator is the measured radius, $R_{T 0}(\Psi)$ is the radius (predicted by Eq. (A1)) and $R_{T 0}^{\text {avg }}=14.34 R_{E}$ is

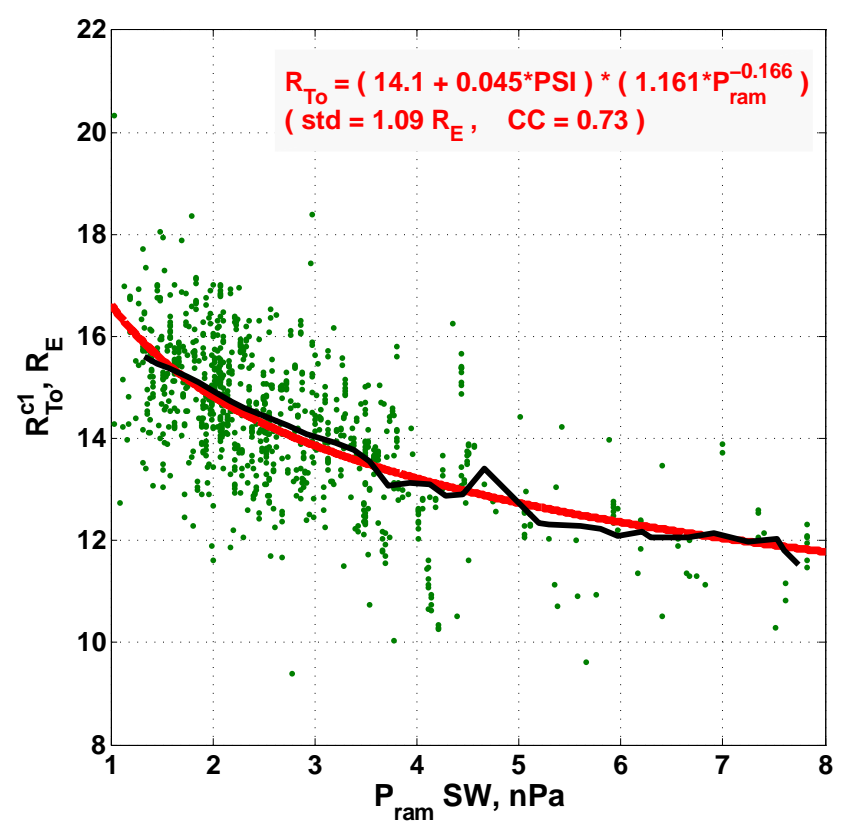

Figure A2. The relationship between solar wind dynamic pressure and terminator radius with subtracted dipole tilt dependence. The red curve is the regression line corresponding to Eq. (A4). The black line is the moving average (with the window size $1 \mathrm{nPa}$, step size $0.2 \mathrm{nPa})$.

the terminator radius averaged over the whole data set. The bottom panel of Fig. A1 visualizes the corrected data with the $\Psi$ dependence subtracted according to Eq. (A2).

Now we can determine the $R_{T 0}$ dependence on solar wind dynamic pressure using refined data $R_{T 0}^{c 1}$. Considering the theoretical background and experience of previous authors, the power law dependence

$R_{T 0}^{c 1}=a P_{\mathrm{d}}^{-b}$,

with constant coefficients $a$ and $b$ is expected (Fig. A2). Taking the logarithm of both sides of Eq. (A3) and applying linear regression we find the coefficients

$R_{T 0}^{c 1}=(16.64 \pm 0.21) P_{\mathrm{d}}^{-0.166 \pm 0.012}$.

Dividing the $R_{T 0}^{c 1}$ by normalization factor $R_{T 0}^{\text {avg }}$ we obtain the explicit dependence

$R_{T 0}=R_{T 0}(\Psi) \cdot\left[(1.161 \pm 0.015) P_{\mathrm{d}}^{-0.166 \pm 0.012}\right]$.

To reveal the IMF $B_{z}$ effect on the $R_{T 0}$, we again construct the normalized correction $R_{T 0}^{c 2}$ with subtracted dependence $R_{T 0}\left(\Psi, P_{\mathrm{d}}\right)$ given by Eq. (A5). The results of regression analysis (Fig. A3) show moderate dependence of corrected $R_{T 0}^{c 2}$ quantity on IMF $B_{z}$ with $\sim 1 R_{E}$ variance amplitude inside the used parameter range. Dividing $R_{T 0}^{c 2}$ by $R_{T 0}^{\mathrm{avg}}$ 


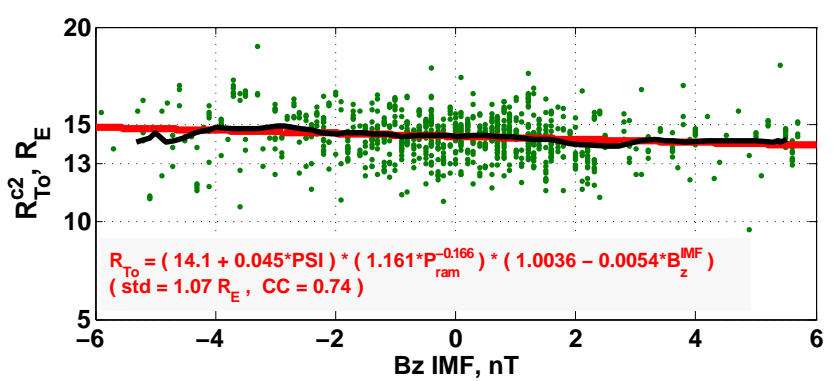

Figure A3. Relationship between IMF $B_{z}$ and terminator radius corrected to eliminate the dipole tilt and ram pressure dependences. Red straight line - regression line. Black line is the moving average (with the window size $2 \mathrm{nT}$, step size $0.2 \mathrm{nT}$ ).

we get

$$
\begin{aligned}
R_{T 0} & =R_{T 0}\left(\Psi, P_{\mathrm{d}}\right) \\
\cdot & {\left[(1.004 \pm 0.005)-(0.0054 \pm 0.0022) \mathrm{IMF} B_{z}\right] . }
\end{aligned}
$$

Substituting the $R_{T 0}\left(\Psi, P_{\mathrm{d}}\right)$ dependence we come to Eq. (7):

$$
\begin{gathered}
R_{T 0}=(14.1+0.045 \Psi) \cdot 1.161 P_{\mathrm{d}}^{-1 / 6.02} \\
\cdot\left(1.004-0.0054 \mathrm{IMF} B_{z}\right)
\end{gathered}
$$

for the radius of the northern terminator magnetopause (radius of the southern side can be obtained by reversing the sign of dipole tilt angle). The resultant correlation coefficient between the predicted terminator radius and that "measured" from the original data set is $C C=0.74$ with the standard deviation $\mathrm{SD}=1.07 R_{E}$, which is significantly better than what PR96 gives $\left(C C_{\mathrm{PR} 96}=0.54, \mathrm{SD}_{\mathrm{PR} 96}=1.27 R_{E}\right)$. To verify the statistical significance of our model we performed the Student's $t$ test (Hudson, 1964) of regression coefficients in Eq. (7) and found that each coefficient undoubtedly satisfies the condition of significance. As an example, the calculated $t$ statistic value $t_{\text {calc }}=4.83$ is most weak for the second coefficient of $R_{T 0}$ (IMF $B_{z}$ ) dependence; however, it is still larger than the critical value even for the highest confidence probability $(\mathrm{cp}=0.999) t_{\text {crit }}=3.3$.

Remember that in order to "move" the original data to the terminator plane we used the analytical magnetopause shape from PR96. To verify how much this procedure could affect the results, we tried another model developed by Shue et al. (1998) [hereafter S98] with another analytical shape and SW/IMF dependence. Results appeared very close: the coefficients $R_{T 0}(\Psi)$ and $R_{T 0}$ (IMF $B_{z}$ ) are almost the same, whereas the coefficients $R_{T 0}\left(P_{\mathrm{d}}\right)$ only slightly differ:

$$
\begin{aligned}
& R_{T 0}(S 98 \text { tracing })= \\
& (14.19+0.046 \Psi) \cdot 1.178 P_{\mathrm{d}}^{-1 / 5.48} \cdot\left(1.004-0.006 \mathrm{IMF} B_{z}\right),
\end{aligned}
$$

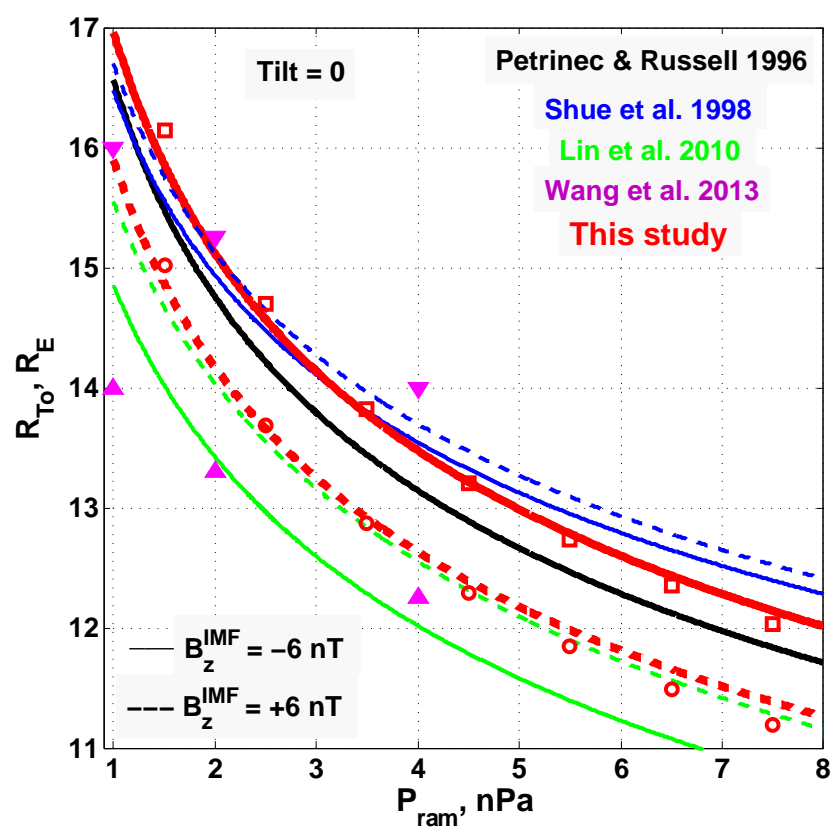

Figure A4. Terminator radius dependence on solar wind dynamic pressure for different empirical models (see the legend). For each model, two profiles corresponding to IMF $B_{z}=+6 \mathrm{nT}$ and IMF $B_{z}=-6 \mathrm{nT}$ are shown. Dipole tilt is zero. Solid and dashed red lines show the results of the new model developed using the PR96 magnetopause shape for tracing to $x=0$. Red empty circles and squares correspond to tracing based on the Shue et al. (1998) magnetopause shape.

confirming validity of the tracing procedure. Results obtained based on PR96 and S98 models are presented in Fig. A4.

\section{A2 Features of the new model}

The developed empirical model of the terminator radius has a series of interesting features. It demonstrates a strong enough perturbation of magnetopause position in the $x=0$ cross section associated with dipole tilting in the $x z$ plane (about $3 R_{E}$ for extreme opposite tilts) in agreement with the modern asymmetric magnetopause models L10 and W13. The coefficient of the power law in dynamic pressure dependence was found to be close to $-1 / 6$ in accordance with the PR96 model.

However, the most interesting observation is the IMF $B_{z}$ dependence of $R_{T 0}$. First of all, we found a clear and statistically significant IMF $B_{z}$ effect (about $1 R_{E}$ ) in contrast to PR96 model. Note that the $R_{T 0}$ (IMF $B_{z}$ ) linear dependence is the same for the negative and positive IMF $B_{z}$ values. It seems that this is not the result of the regression method but is a real feature supported by the moving average values (Fig. A3). Our results demonstrate the increase of the terminator radius for negative IMF $B_{z}$ and its decrease for positive IMF $B_{z}$. Since the cusp axis is almost always located sun- 
ward of the $x=0$ plane (except during extreme dipole tilt angles), such dependence looks natural. During southward IMF $B_{z}$, the magnetic tubes, reconnected on the dayside, convect in the anti-sunward direction and drape the magnetopause tailward of the cusps, increasing the radius at terminator. The picture is the opposite for the northward IMF - the solar wind tubes reconnect behind the cusps adding magnetic flux to the dayside magnetosphere and reducing the magnetic flux and radius tailward of the cusps including the terminator plane. However, it should be noted that the L10 and S98 models predict the opposite $R_{T 0}\left(\mathrm{IMF} B_{z}\right.$ ) dependence. This discrepancy is likely due to the analytical predefinition of the magnetopause shape in those models which turns out to be a limitation not only in the quantitative but also in the qualitative sense. The most recent 3-D magnetopause model W13 was constructed using an excellent set of crossings without any assumptions about the analytical shape. This model predicts the same qualitative terminator radius dependence on IMF $B_{z}$ as the present study (Fig. A4) with more pronounced (roughly by factor 2) difference of $R_{T 0}$ between southward and northward IMF orientation. However, W13 has no explicit analytical parametrization which makes its application difficult. Several points that we used for comparison in Fig. A4 were adopted from their Fig. 10. It is also worth noting that the same sign of the $R_{T 0}$ (IMF $B_{z}$ ) dependence was obtained for the BATS-R-US "empirical" magnetopause model (Lu et al., 2013). This conformity with our new formula could be the main reason for the estimation improvement described in Sect. 4. 
Acknowledgements. We thank V. A. Sergeev for useful discussions and Marianna Holeva for her help in the manuscript preparation. We are grateful to the topical editor C. Owen for their objective assessment of this paper and to the referees for their constructive criticism.

Global MHD simulation results were provided by the Community Coordinated Modeling Center at the Goddard Space Flight Center through their public Runs on Request system (http://ccmc. gsfc.nasa.gov/). The BATS-R-US Model was developed by the group led by Tamas Gombosi at the Center for Space Environment Modeling, University of Michigan.

The work was carried out as part of EU FP7 ECLAT project. Data on magnetotail magnetic flux ( $F_{1}$ and $F_{2}$ values) can be found on the site http://geo.phys.spbu.ru/eclat/. E. Gordeev's work was supported by a grant from the Government of St. Petersburg, Russian Federation (2014).

The topical editor C. Owen thanks L. B. N. C. Clausen and the two anonymous referees for help in evaluating this paper.

\section{References}

Boyle, C. B., Reiff, P. H., and Harrison, M. R.: Empirical polar cap potentials, J. Geophys. Res., 102, 111, doi:10.1029/96JA01742, 1997.

Brittnacher, M., Fillingim, M., Parks, G., Germany, G., and Spann, J.: Polar cap area and boundary motion during substorms, J. Geophys. Res., 104, 12251-12262, doi:10.1029/1998JA900097, 1999.

Clausen, L. B. N., Baker, J. B. H., Ruohoniemi, J. M., Milan, S. E., and Anderson, B. J.: Dynamics of the region 1 Birkeland current oval derived from the Active Magnetosphere and Planetary Electrodynamics Response Experiment (AMPERE), J. Geophys. Res., 117, A06233, doi:10.1029/2012JA017666, 2012.

DeJong, A. and C. Clauer: Polar UVI images to study steady magnetospheric convection events: Initial results, Geophys. Res. Lett., 32, L24101, doi:10.1029/2005GL024498, 2005.

Fairfield, D. H., and J. Jones: Variability of the tail lobe field strength, J. Geophys. Res.,101(A4), 7785-7791, doi:10.1029/95JA03713, 1996.

Gordeev, E., Facskó, G., Sergeev, V., Honkonen, I., Palmroth, M., Janhunen, P., and Milan, S.: Verification of the GUMICS-4 global MHD code using empirical relationships, J. Geophys. Res. Space Physics, 118, 3138-3146, doi:10.1002/jgra.50359, 2013.

Gordeev, E. I., Sergeev, V. A., Pulkkinen, T. I., and Palmroth, M.: Contribution of magnetotail reconnection to the cross-polar cap electric potential drop, J. Geophys. Res., 116, A08219, doi:10.1029/2011JA016609, 2011.

Hubert, B., Milan, S. E., Grocott, A., Cowley, S. W. H., and Gerard, J.-C.: Dayside ang nightside reconnection rates inferred from IMAGE-FUV and SuperDARN data, J. Geophys. Res., 111, A03217, doi:10.1029/2005JA011140, 2006.

Hudson, D. J.: Lectures on elementary statistics and probability, Geneva, Switzerland, 1964.
Kaymaz, Z., Siscoe, G., Luhmann, J. G., Fedder, J. A., and Lyon, J. G.: Interplanetary magnetic field control of magnetotail field: IMP 8 data and MHD model compared, J. Geophys. Res., 100, 17163-17172, doi:10.1029/95JA00593, 1995.

Lin, R. L., Zhang, X. X., Liu, S. Q., Wang, Y. L., and Gong, J. C.: A three-dimensional asymmetric magnetopause model, J. Geophys. Res., 115, A04207, doi:10.1029/2009JA014235, 2010.

Lu, J. Y., Liu, Z.-Q., Kabin, K., Jing, H., Zhao, M. X., and Wang, Y.: The IMF dependence of the magnetopause from global MHD simulations, J. Geophys. Res., 118, 3113-3125, doi:10.1002/jgra.50324, 2013.

Milan, S. E., Lester, M., Cowley, S. W. H., Oksavik, K., Brittnacher, M., Greenwald, R. A., Sofko, G., and Villain, J.-P.: Variations in the polar cap area during two substorm cycles, Ann. Geophys., 21, 1121-1140, doi:10.5194/angeo-21-1121-2003, 2003.

Palmroth, M., Pulkkinen, T. I., Janhunen, P., and Wu, C.-C.: Stormtime energy transfer in global MHD simulation, J. Geophys. Res., 108, 1048, doi:10.1029/2002JA009446, 2003.

Petrinec, S. M. and Russell, C. T.: Near-Earth magnetotail's hape and size as determined from the magnetopause flaring angle, $\mathrm{J}$. Geophys. Res., 101, 137-152, doi:10.1029/95JA02834, 1996.

Powell, K. G., Roe, P. L., Linde, T. J., Gombosi, T. I., and de Zeeuw, D. L.: A solution-adaptive upwind scheme for ideal magnetohydrodynamics, J. Comput. Phys., 154, 284-309, doi:10.1006/jcph.1999.6299, 1999.

Raeder, J., Larson, D., Li, W., Kepko, L., and Fuller-Rowell, T.: OpenGGCM simulations for the THEMIS mission, Space Sci. Rev., 141, 535-555, doi:10.1007/s11214-008-9421-5, 2008.

Russell, C. T. and McPherron, R. L.: The magnetotail and substorms, Space Sci. Rev., 15, 205-266, doi:10.1007/BF00169321, 1973.

Shue, J.-H., Chao, J. K., Fu, H. C., Khurana, K. K., Russell, C. T., Singer, H. J., and Song, P.: Magnetopause location under extreme solar wind conditions, J. Geophys. Res., 103, 1769117700, doi:10.1029/98JA01103, 1998.

Shukhtina, M. A., Dmitrieva, N. P., and Sergeev, V. A.: Quantitative magnetotail characteristics of different magnetospheric states, Ann. Geophys., 22, 1019-1032, doi:10.5194/angeo-221019-2004, 2004.

Shukhtina, M. A., Gordeev, E. I., and Sergeev, V. A.: Time-varying magnetotail magnetic flux calculation: a test of the method, Ann. Geophys., 27, 1583-1591, doi:10.5194/angeo-27-15832009, 2009.

Shukhtina, M. A., Sergeev, V. A., DeJong, A. D., and $\mathrm{Hu}-$ bert, B.: Comparison of magnetotail magnetic flux estimates based on global auroral images and simultaneous solar windmagnetotail measurements, J. Atmos. Sol.-Terr. Phy., 72, 1282 1291, doi:10.1029/98JA01103, 2010.

Wang, Y., Sibeck, D. G., Merka, J., Boardsen, S. A., Karimabadi, H., Sipes, T. B., Šafránková, J., Jelínek, K., and Lin, R.: A new three-dimensional magnetopause model with a support vector regression machine and a large database of multiple spacecraft observations, J. Geophys. Res.-Space, 118, 2173-2184, doi:10.1002/jgra.50226, 2013. 